\title{
6.8 Static and dynamic properties of patterned magnetic permalloy films
}

\author{
B. Hillebrands, C. Mathieu, C. Hartmann, M. Bauer, O. Büttner, S. Riedling, B. Roos, and \\ S.O. Demokritov ${ }^{1}$
}

The physical properties of magnetic particles on the micro- and nanometer scale have attracted much interest in the last few years especially because of the promising prospects in future data storage and sensor applications [1-6]. There remain many unresolved problems in the preparation and characterization of these structures, and therefore suitable preparation procedures and analysis tools are still under development. It is of particular importance to develop fabrication methods for magnetic islands with a very narrow distribution of the magnetic parameters and with well controlled coupling between the islands, which for many applications should be made negligibly small. In particular a precise control of magnetic anisotropies induced by the patterning process as well as by finite size effects is required. Here we report on studies of regular square arrays of magnetic dots by Brillouin light scattering (BLS), Kerr magnetometry and magnetic force microscopy. From the measured spin wave dispersion properties obtained from the BLS experiments the uniformity of the magnetization distribution, demagnetizing effects as well as coupling strengths between magnetic dots are obtained.

Well separated dots of circular shape made of polycrystalline permalloy $\left(\mathrm{Ni}_{80} \mathrm{Fe}_{20}\right)$ will not exhibit any in-plane anisotropy. For dots with small enough separation any observed in-plane anisotropy is therefore a fingerprint for anisotropic, magnetic coupling between the dots. Measurements of coupling induced anisotropies allow for a quantitative study of magnetic interdot coupling.

The method of choice is Brillouin light scattering from dipolar dominated spin wave modes (DamonEshbach modes) propagating within the dots [7]. The spin wave frequencies depend on the demagnetization factor of each dot, on the value and distribution of the magnetization vector, on anisotropies, and to a weak degree on the volume exchange constant. A study of the mode dispersion and of the dependence on the in-plane direction of the external field allows to determine the interdot coupling strength.

We have studied regular square lattices of circular shaped dots of permalloy of $1 \mu \mathrm{m}$ and $2 \mu \mathrm{m}$ diameter and $500 \AA$ and $1000 \AA$ thickness. The films were prepared in ultrahigh vacuum onto $\mathrm{Si} / \mathrm{SiO}_{2}$ 10x10 $\mathrm{mm}^{2}$ substrates using an $\mathrm{e}^{-}$-beam evaporator. The samples were patterned by synchrotron radiation based X-ray lithography performed at the L2M synchrotron station at the super ACO storage at LURE, Orsay, using ion beam etching to transfer the patterns into the permalloy films. A subtractive process was used as described elsewhere [8]. Each sample consists of circular dots arranged in a $1 \times 1 \mathrm{~mm}^{2}$ square lattice with a diameter/periodicity of 1/1.1, 1/2, 2/2.2 and 2/4 $\mu \mathrm{m}$, respectively. For comparison, and in order to check for modifications of the magnetic bulk properties during the patterning process, the remaining parts of the samples were not patterned, i.e. consist of a continuous film. Depth profile measurements show that the etching process cuts $200 \AA$ deep into the substrate between the dots, which guarantees that $\mathrm{Ni}_{80} \mathrm{Fe}_{20}$ bridges between the dots do not exist. Electron micrographs show as well that neighboring dots are not touching.

The Brillouin light scattering experiments were performed in backscattering geometry using a digitally controlled, fully automated multipass tandem Fabry-Perot interferometer with the in-plane mo-

\footnotetext{
${ }^{1}$ in collaboration with B. Bartenlian, and C. Chappert, IEF, Université Paris Sud, 91405 Orsay, France, D. Decanini, F. Rousseaux, and E. Cambril, L2M, Bagneux, France A. Müller, B. Hoffmann, and U. Hartmann, Universität Saarbrücken, Germany
} 
mentum transfer perpendicular to the applied magnetic field. $\mathrm{Ar}^{+}$-ion laser light with an incident power of at most $100 \mathrm{~mW}$ and an angle of incidence of $45^{\circ}$ degrees was focused onto the sample with a focus diameter of about $40 \mu \mathrm{m}$. The spin wave frequencies were detected as a function of the strength, $H$, and the angle, $\Phi_{\mathrm{H}}$, of the in-plane applied magnetic field.

The magnetization reversal behavior as well as the zero field domain structure have been investigated using Kerr magnetometry and magnetic force microscopy. Kerr loops have been measured as a function of the angle $\Phi_{\mathrm{H}}$ of the in-plane applied field. The focus diameter of the probing laser light was below $0.3 \mathrm{~mm}$ to ensure alignment within the patterned area. Magnetic force microscope images have been made at zero applied field using a Co coated Si-cantilever at a sample distance between 10 and $100 \mathrm{~nm}$ at a vibration frequency of $69.62 \mathrm{kHz}$. The spatial resolution is below $100 \mathrm{~nm}$.

A detailed BLS study of the dependence of the spin wave properties on the applied magnetic field has been performed. Fig. 1 shows a typical Brillouin light scattering spectrum. The scattering geometry is shown in the inset of Fig. 2. At zero frequency the elastically scattered light, and at frequencies of $\pm(33-37) \mathrm{GHz}$ the ghost peaks are observed. In the regions of $\pm(6.5-22) \mathrm{GHz}$ the inelastically scattered signal is recorded. We observe the Damon-Eshbach mode, the first standing spin wave (volume mode) and a surface phonon (see Fig. 1). The obtained spin wave dispersion is shown in Fig. 2 for the $2 / 2.2 \mu \mathrm{m}$ sample.

With negligibly small anisotropies [9], as obtained for the permalloy film, the frequency of the Damon-Eshbach mode can be expressed as [7]

$$
v=\gamma \cdot\left[H_{i} \cdot\left(H_{i}+4 \pi M_{s}\right)+\left(2 \pi M_{s}\right)^{2} \cdot\left(1-e^{-2 q_{\|} d}\right)\right]^{1 / 2}
$$

with $\gamma$ the gyromagnetic ratio, $4 \pi M_{\mathrm{s}}$ the saturation magnetization, $q_{\|}$the in-plane wave vector, and $d$ the dot thickness. $H_{\mathrm{i}}$ is the internal field, which is here defined as

$$
H_{i}=H-H_{d}=H-N \cdot 4 \pi M_{s}
$$

with $N$ the demagnetizing factor of the magnetic dots and $H_{\mathrm{d}}$ the demagnetizing field. Eq. (1) presumes a saturation of in-plane magnetization of the dots.

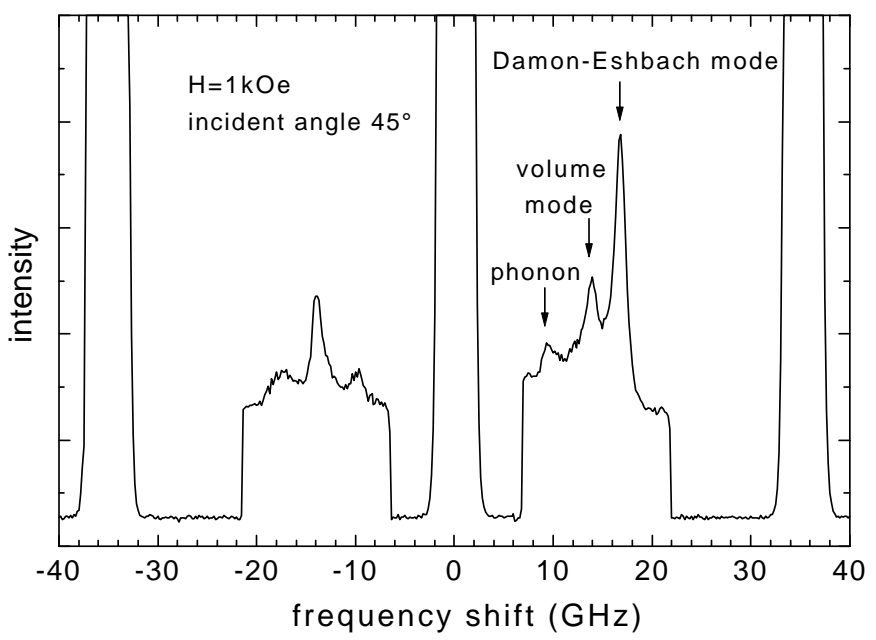

Fig. 1. Spin wave spectrum of a $2 / 4 \mu \mathrm{m}$ sample of $500 \AA$ thickness at an applied field of $1 \mathrm{kOe}$ at an angle of light incidence of $45^{\circ}$. At zero frequency the elastically scattered light, and at frequencies of $\pm(33-37) \mathrm{GHz}$ the first ghost peaks are observed. In the region of interest $\pm(6.5-22) \mathrm{GHz}$ the scan speed is reduced and thus the scattering intensity is increased. As indicated in the Figure the DamonEshbach mode, a standing spin wave (volume mode) and a surface phonon is observed.

Fig. 2 shows the observed dispersion curve of the Damon-Eshbach mode (squares) for the $2 / 2.2 \mu \mathrm{m}$ sample as a function of the in-plane wave vector, $q_{\|}$. The solid line is a fit using Eq. (1) with the thickness $d$ of the dots as a fit parameter. The fitted value of $d=940 \AA$ is in very good agreement 
with the nominal thickness of $1000 \AA$. No indications of a mode quantization due to the finite size of the dots, i.e., a lateral confinement of the spin wave modes, as reported for magnetic bars by Gurney et al. [2], or of spin wave zone folding effects as predicted by Kolodin et al. [10] have been observed for the investigated samples.

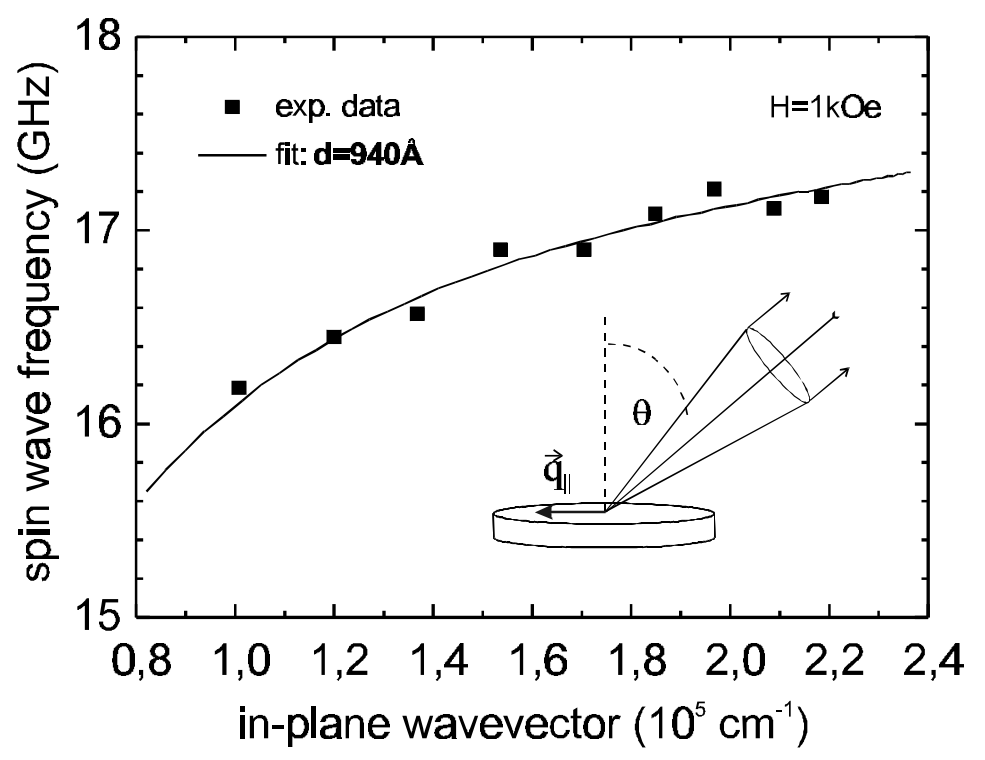

Fig. 2. Dispersion of the spin wave frequency for the $2 / 2.2 \mu \mathrm{m}$ sample as a function of the in-plane wave vector, $q_{\|}$. The scattering geometry is indicated in the inset. The squares are the experimental data and the solid line is a fit to the data.

We have investigated in detail the dependence of the spin wave frequencies on the strength of the external field [5,6]. Fig. 3 shows the results for the $1000 \AA$ thick films. As obtained from the data displayed in Fig. 3 the spin wave frequencies are mostly sensitive to the dot diameter and to a much lesser degree to the dot separation. The solid lines are model calculations using Eq. (1) and calculating the demagnetizing factor, $N$, assuming spheroidally shaped dots with diameter $l$ to thickness $d$ ratios as indicated in the figure. A fairly good agreement between the measured and the calculated values is achieved for the data of spin wave frequencies larger than $14.5 \mathrm{GHz}$, where the dots are magnetically saturated. For smaller values, i.e., for $H<1 \mathrm{kOe}$ for the $l=0.5 \mu \mathrm{m}$ dots, and $H<0.6$ $\mathrm{kOe}$ for the $l=1.0 \mu \mathrm{m}$ dots, a breakdown of a uniform island magnetization is observed.

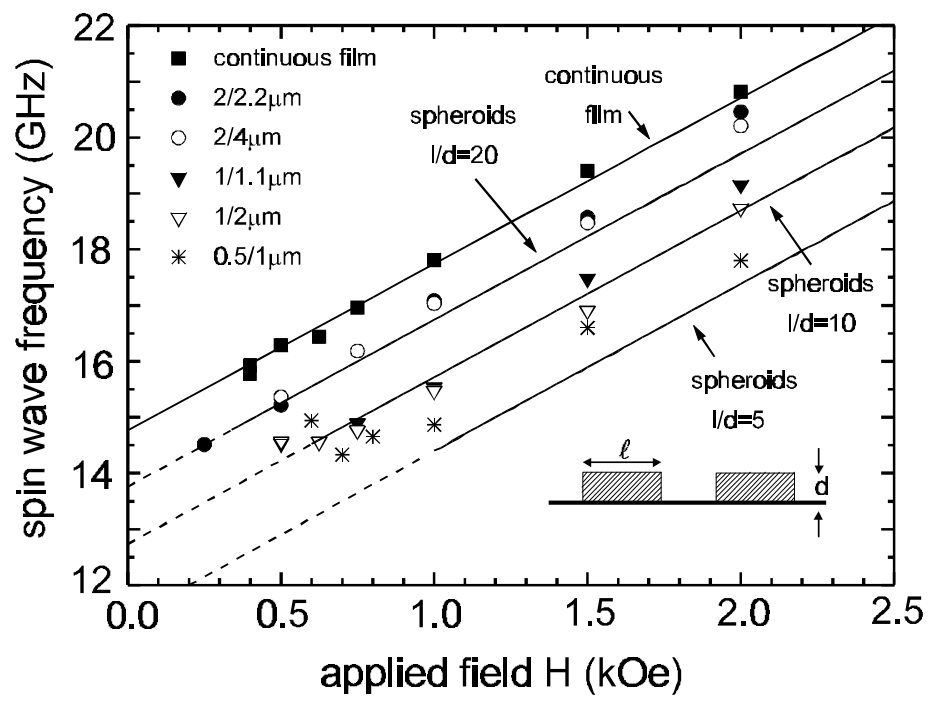

Fig. 3. Measured spin wave frequencies as a function of the strength of the applied external field, $H$. The symbols denote the measured data as indicated. The solid lines are fits to the data using aspect ratios of the spheroidal dot shapes as indicated in the Figure.

To clarify the problem of an in-plane interdot coupling, we measured the spin wave frequencies of the Damon-Eshbach mode as a function of the angle of the in-plane applied field, $\Phi_{\mathrm{H}}$, with respect to a reference [10]-direction of the lattice. Only for the smallest dot separations of $0.1 \mu \mathrm{m}$ a fourfold 
anisotropic behavior is found. This is displayed in Fig. 4 for the 1/1.1 $\mu \mathrm{m}$ and, for comparison, for the $1 / 2 \mu \mathrm{m}$ lattices of the sample of $1000 \AA$ thickness at an applied field of $1 \mathrm{kOe}$ [11]. To determine quantitatively the anisotropy constant we use the free anisotropy energy expression

$$
F=K^{(4)} \sin ^{2} \Phi \cdot \cos ^{2} \Phi
$$

with $\Phi$ the angle between the direction of magnetization with respect to the [10]-direction, and $K^{(4)}$ a fourfold in-plane anisotropy constant

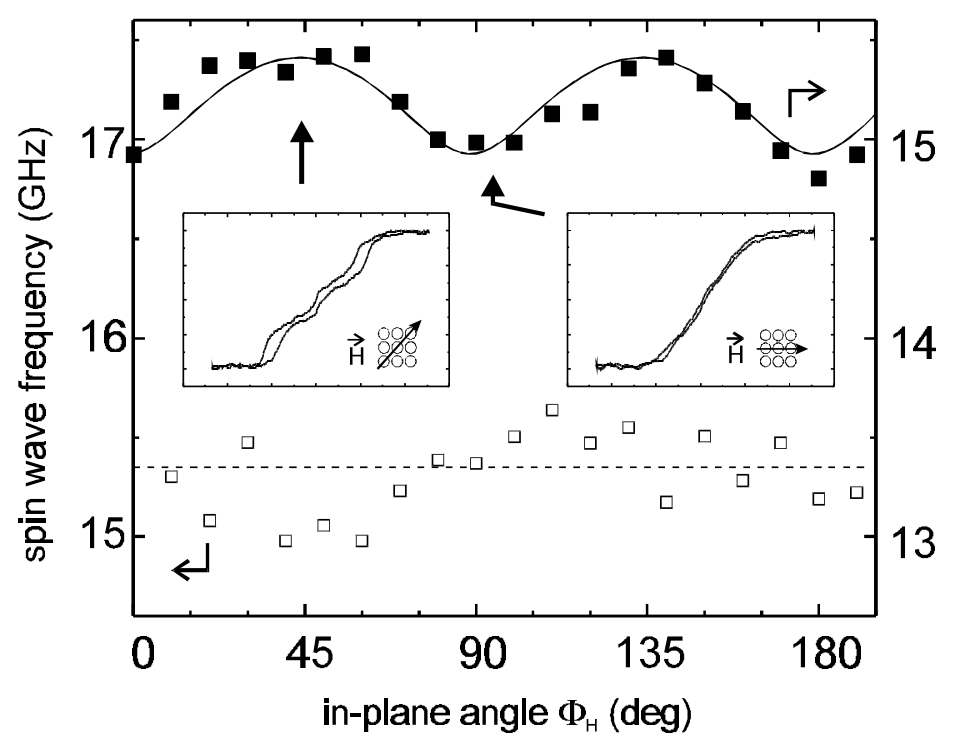

Fig. 4. Dependence of the spin wave frequencies on the in-plane direction of the applied field for the $1 / 1.1 \mu \mathrm{m}(\boldsymbol{\square})$ and, for comparison, for the $1 / 2$ $\mu \mathrm{m}$ ( $\square)$ dot lattices of $1000 \AA$ thickness. The solid line is a fit for the data (see text). Kerr hysteresis loops of the $1 / 1.1 \mu \mathrm{m}$ dot lattice are shown as insets with the applied field as indicated. Note the complicated magnetization reversal behavior of the $1 / 1.1 \mu \mathrm{m}$ dot lattice for a field direction of $45^{\circ}$.

A model fit using Eq. (3) and a numerical procedure to calculate the spinwave frequencies [12] is displayed in Fig. 4 as a solid line for the $1 / 1.1 \mu \mathrm{m}$ lattice. For both $1 / 1.1 \mu \mathrm{m}$ samples with thicknesses of $500 \AA$ and $1000 \AA$ the anisotropy contribution $K^{(4)}$ was determined for several values of the applied magnetic field strength, $H$. The obtained results for $K^{(4)}$ are displayed for both thicknesses in Fig. 5 as a function of the reduced field $H / H_{\mathrm{d}} . K^{(4)}$ decreases with increasing reduced field and saturates within the investigated field range at $K^{(4)}=-0.6 \cdot 10^{5} \mathrm{erg} / \mathrm{cm}^{3}$, which corresponds to an effective anisotropy field $H_{\mathrm{ani}}=150 \mathrm{Oe}$, at the same reduced field value of about $H / H_{\mathrm{d}}=5$. The data of both dot thicknesses fall onto one common curve within the error margins indicating that the coupling strength scales with the demagnetizing field.

The origin of this fourfold anisotropy can be understood as follows. Because of the large distance of $0.1 \mu \mathrm{m}$ between the dots a direct exchange mechanism via conduction electrons or via electron tunneling can be excluded. The quasi-uniform demagnetization field, caused by the shape of the patterned area $\left(1 \mathrm{x} 1 \mathrm{~mm}^{2}\right.$ square) can not be responsible for the observed anisotropy. In fact, the film thickness/square length ratio is of the order of $10^{-4}$. It corresponds to a demagnetizing field of about $1 \mathrm{Oe}$, which is much smaller than the observed value of $150 \mathrm{Oe}$. Moreover, since the quasi-uniform field strength should depend on the average magnetization of the lattice, the effect should be the same for $1 / 1.1 \mu \mathrm{m}$ and the $2 / 2.2 \mu \mathrm{m}$ lattices, which is not the case. A dipolar interaction of completely magnetized dots also cannot account for the observed anisotropy, because the corresponding dipolar energy can be expressed as a bilinear form of the components of the magnetization vector. Such an expression can only yield an uniaxial, but not a four-fold anisotropy contribution, since in a bilinear form the direction cosines appear quadratic in highest order and add to a constant if a fourfold symmetry is given. Therefore we propose that the fourfold anisotropy contribution is caused by a dipole-dipole interaction between residual unsaturated parts of the dots, because in this case the 
energy cannot be expressed as a bilinear form. The large observed decrease of the coupling anisotropy constant with increasing field as well as the scaling of the anisotropy with the reduced field corroborates this assumption. The anisotropic coupling is still observed for field values larger than the demagnetizing field, $H_{\mathrm{d}}$. However, due to the non-ellipsoidal form of the dots and due to surface magnetic anisotropies acting on the dot boundaries, which cannot be characterized in more detail in the present study, it is likely that the non-saturated parts can persist up to fairly high fields.

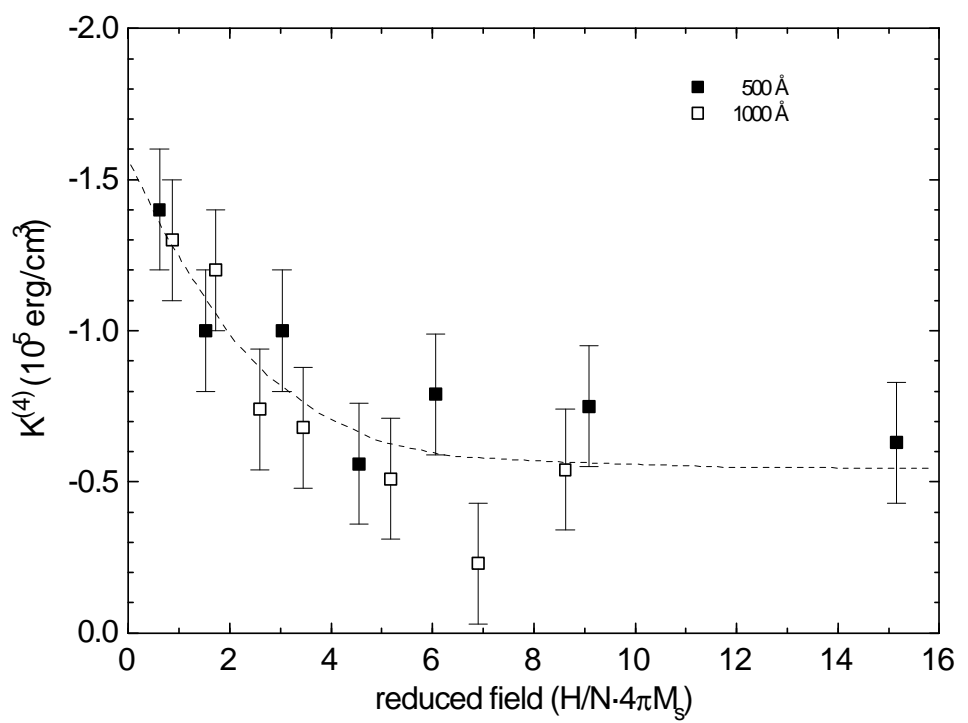

Fig. 5. Fourfold anisotropy constant, $K^{(4)}$, for the $1 / 1.1 \mu \mathrm{m}$ lattices of $500 \AA$ and $1000 \AA$ thickness as a function of the reduced field $\left(H / N \cdot 4 \pi M_{\mathrm{s}}\right)$. The dashed line serves as a guide to the eyes.

To study more precisely the static magnetization behavior Kerr measurements were carried out. Only for the lattices with the smallest dot separation of $0.1 \mu \mathrm{m}$ a fourfold in-plane anisotropy in the loop shapes is found. For comparison Kerr loops of the $1 / 1.1 \mu \mathrm{m}$ lattice of $1000 \AA$ thickness are shown as insets in Fig. 4, with the magnetic field, $H$, applied along the [10]- and the [11]-direction. In comparison, the loop shape of the $1 / 1.1 \mu \mathrm{m}$ lattice along the [10]-direction is very similar to the loop shapes of the $1 / 2 \mu \mathrm{m}$ lattices for all field directions. In contrary the loop shape of the $1 / 1.1 \mu \mathrm{m}$ lattice with $H$ along the [11]-direction, i.e. the easy direction of $K^{(4)}$ as determined by BLS, is rather complex. Similar loop shapes have been obtained by Smyth et al. as a function of the interdot separation but the magnetization reversal process is not yet understood [1,3]. Since these loop shapes exhibit a higher zero-field susceptibility, they also indicate that the [11]-direction is the easy axis. Another result is that the saturation field $H_{\mathrm{s}}$ decreases with decreasing dot separation, which can be well understood as a result of an increasing film coverage. For the $1 / 1.1 \mu \mathrm{m}$ and the $1 / 2 \mu \mathrm{m}$ lattices the values of $H_{s}$ are $(620 \pm 50)$ Oe and $(780 \pm 50)$ Oe, respectively. This result is qualitatively in agreement with the BLS data, showing the spin wave frequencies of the $1 / 1.1 \mu \mathrm{m}$ lattice lying slightly above the frequencies of the $1 / 2 \mu \mathrm{m}$ indicating smaller demagnetizing fields for the sample with lower spacings. A direct proof of a coupling of stray fields due to the presence of a common magnetic substructure of the dots is yielded by magnetic force microscopy (MFM). The measurements were performed at zero applied field. MFM-images of the $1 / 1.1 \mu \mathrm{m}$ and the $1 / 2 \mu \mathrm{m}$ lattices of $500 \AA$ thickness are shown in Fig. 6. For both lattices a heterogeneous magnetic structure is clearly recognizable, which seems to be totally disordered for the large separation of $1 \mu \mathrm{m}$. For the 1/1.1 $\mu \mathrm{m}$ lattice a regular pattern of the domain walls can be seen, which are aligned crosslike along the [10]-direction, consistent with a closed domain structure. This is evidence for a separation dependent dipolar coupling of the domains, which is only effective for separations of the order of $0.1 \mu \mathrm{m}$. 

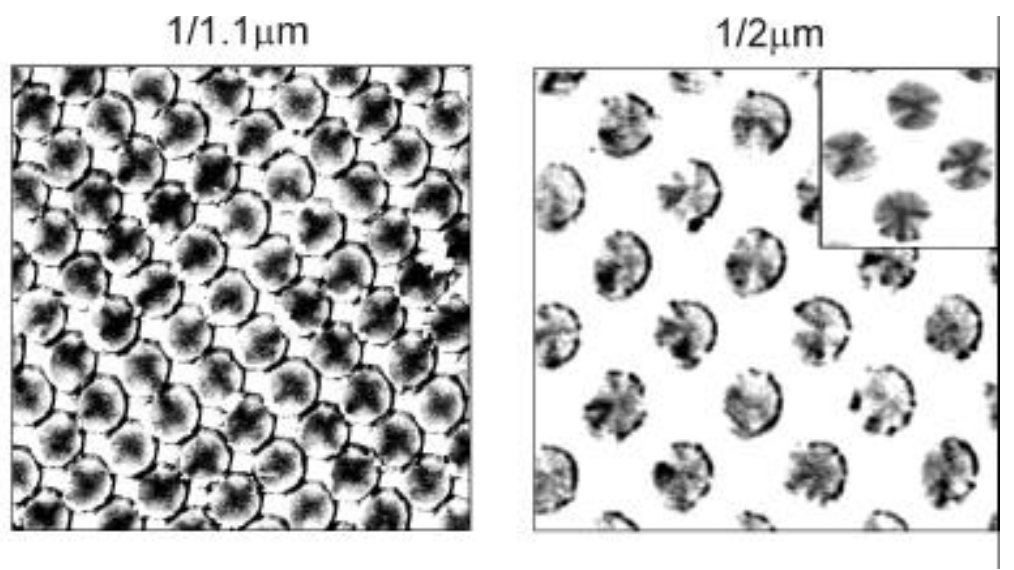

Fig. 6. MFM images of the $1 / 1.1 \mu \mathrm{m}$ and $1 / 2 \mu \mathrm{m}$ samples of $500 \AA$ dot thickness. Note the crosslike order of domain walls along the [10]-direction for the $1 / 1.1 \mu \mathrm{m}$ lattice in contrary to their irregular orientation for the $1 / 2 \mu \mathrm{m}$ lattice. For clarity an inset with a better resolution is shown for the $1 / 2 \mu \mathrm{m}$ sample.

In summary, using spin waves as a tool to investigate magnetic properties in dot arrays, the most striking effect is the demagnetizing effect of the individual dots. It should be noted that the large dependence on the demagnetizing factor is due to the fact, that the spin wave wavelength of $\approx 2000$ $\AA$ is comparable to the island sizes. A breakdown of the uniform island magnetization can be easily observed in the spin wave spectra. From fitting the spin wave dispersion curves the dot thicknesses obtained agree with the nominal thicknesses. We have found clear evidence for a fourfold in-plane anisotropic coupling of magnetic micron dots, most probably mediated by stray fields of nonsaturated parts of the dots. The anisotropy strength is of the order of $10^{5} \mathrm{erg} / \mathrm{cm}^{3}$. Since the coupling has been found only for the smallest dot separation of $0.1 \mu \mathrm{m}$ investigations of further reduced dot separations are required. For a full description of the spin wave mode dispersion in these patterned structures model calculations taking in detail the size and the shapes of the islands into account are greatly needed.

\section{References}

[1] J.F. Smyth, S. Schultz, D. Kern, H. Schmidt, D. Yee, J. Appl. Phys. 63, 4237, (1988).

[2] B.A. Gurney, P. Baumgart, V. Speriosu, R. Fontana, A. Patlac, T. Logan, P. Humbert, Digest of the International Conference on Magnetic Films and Surfaces, Glasgow, (1991).

[3] J.F. Smyth, S. Schultz, D.R. Fredkin, D.P. Kern, S.A. Rishton, H. Schmid, M. Cali, T.R. Koehler, J. Appl. Phys. 69, 5262 (1991).

[4] A. Maeda, M. Kume, T. Ogura, K. Kukori, T. Yamada, M. Nishikawa, Y. Harada, J. Appl. Phys. 76, 6667 (1994).

[5] B. Hillebrands, C. Mathieu, M. Bauer, S.O. Demokritov,B. Bartenlian, C. Chappert, D. Decanini, F. Rousseaux, F. Carcenac, J. Appl. Phys. 81 (8) 4993 (1997)

[6] C. Mathieu, C. Hartmann, M. Bauer, O. Büttner, S. Riedling, B. Roos, S.O. Demokritov, B. Hillebrands, B. Bartenlian, C. Chappert, D. Decanini, F. Rousseaux, E. Cambril, A. Müller, B. Hoffmann, and U. Hartmann, Appl. Phys. Lett. 702912 (1997).

[7] R.W. Damon, J.R. Eshbach, J. Phys. Chem. Solids 19, 308 (1961).

[8] F. Rousseaux, D. Decanini, F. Carcenac, E. Cambril, M.F. Ravet, C. Chappert, N. Bardou, B. Bartenlian, P. Veillet, J. Vac. Technol. B 13, 2787 (1995).

[9] Equations $(1,2)$ describe in good approximation the observed gross feature of the shape effects also in presence of anisotropies, since the anisotropy contributions are small.

[10] P.A. Kolodin, B. Hillebrands, J. Magn. Magn. Mater. 161, 199 (1996).

[11] The data of the $1 / 2 \mu \mathrm{m}$ sample show compared to the $1 / 1.1 \mu \mathrm{m}$ sample a larger scatter in the data due to the smaller light scattering cross section caused by the lower magnetic coverage, but the data indicate clearly, that no significant fourfold anisotropy contribution is present in this sample.

[11] B. Hillebrands, Phys. Rev. B 44, 530 (1990). 